\title{
Study of TeV Neutrinos with Upward Showering Muons in Super-Kamiokande
}

\author{
S. Desai ${ }^{\text {c,1,* }}$ K. Abe ${ }^{\text {a }}$ Y. Hayato ${ }^{a}$ K. Iida ${ }^{a}$ K. Ishihara ${ }^{a}$
}

J. Kameda ${ }^{\text {a }}$ Y. Koshio ${ }^{a}$ A. Minamino ${ }^{a}$ C. Mitsuda ${ }^{a}$ M. Miura ${ }^{a}$ S. Moriyama ${ }^{\text {a }}$ M. Nakahata ${ }^{\text {a }}$ Y. Obayashi ${ }^{\text {a }}$ H. Ogawa ${ }^{a}$

M. Shiozawa ${ }^{\text {a }}$ Y. Suzuki ${ }^{\text {a }}$ A. Takeda ${ }^{\text {a }}$ Y. Takeuchi ${ }^{\text {a }}$

K. Ueshima ${ }^{a}$ H. Watanabe ${ }^{a}$ S. Yamada ${ }^{a}$ I. Higuchi ${ }^{b}$

C. Ishihara ${ }^{b}$ M. Ishitsuka ${ }^{b}$ T. Kajita ${ }^{b}$ K. Kaneyuki ${ }^{b}$

G. Mitsuka ${ }^{b}$ S. Nakayama ${ }^{b}$ H. Nishino ${ }^{b}$ K. Okumura ${ }^{b}$ C. Saji ${ }^{b}$

Y. Takenaga ${ }^{\text {b S.T. } \text { Clark }^{c} \text { F. Dufour }}{ }^{c}$ E. Kearns ${ }^{c}$ S. Likhoded ${ }^{c}$

J.L. Raaf ${ }^{c}$ J.L. Stone ${ }^{c}$ L.R. Sulak ${ }^{c}$ W. Wang ${ }^{c}$ M. Goldhaber ${ }^{d}$

D. Casper ${ }^{\text {e }}$ J.P. Cravens ${ }^{\text {e }}$ J. Dunmore ${ }^{\mathrm{e}}$ W.R. Kropp ${ }^{\mathrm{e}}$

D.W. Liu ${ }^{\text {e }}$ S. Mine ${ }^{\mathrm{e}}$ C. Regis ${ }^{\mathrm{e}}$ M.B. Smy ${ }^{\mathrm{e}}$ H.W. Sobel ${ }^{\mathrm{e}}$

M.R. Vagins ${ }^{e}$ K.S. Ganezer ${ }^{f}$ B. Hartfiel ${ }^{f}$ J. Hill ${ }^{f}$ W.E. Keig ${ }^{f}$

J.S. Jang ${ }^{g}$ I.S. Jeong ${ }^{g}$ J.Y. Kim ${ }^{g}$ I.T. Lim ${ }^{g}$ M. Fechner ${ }^{\mathrm{h}}$

K. Scholberg ${ }^{h}$ N. Tanimoto ${ }^{h}$ C.W. Walter ${ }^{h}$ R. Wendell ${ }^{h}$

S. Tasaka ${ }^{i}$ G. Guillian ${ }^{j}$ J.G. Learned ${ }^{j}$ S. Matsuno ${ }^{j}$

M.D. Messier ${ }^{k}$ A. K. Ichikawa ${ }^{\ell}$ T. Ishida ${ }^{\ell}$ T. Ishii ${ }^{\ell}$

T. Kobayashi ${ }^{\ell}$ T. Nakadaira ${ }^{\ell}$ K. Nakamura ${ }^{\ell}$ K. Nitta $^{\ell}$

Y. Oyama ${ }^{\ell}$ Y. Totsuka $^{\ell}$ A.T. Suzuki ${ }^{m}$ M. Hasegawa ${ }^{\mathrm{n}}$

K. Hiraide ${ }^{n}$ I. Kato ${ }^{n}$ H. Maesaka ${ }^{n}$ T. Nakaya ${ }^{n}$ K. Nishikawa ${ }^{n}$

T. Sasaki ${ }^{n}$ H. Sato ${ }^{n}$ S. Yamamoto ${ }^{n}$ M. Yokoyama ${ }^{n}$

T.J. Haines ${ }^{\circ}$ S. Dazeley ${ }^{p}$ S. Hatakeyama ${ }^{p}$ R. Svoboda ${ }^{p}$

M. Swanson ${ }^{\mathrm{q}}$ A. Clough ${ }^{\mathrm{r}}$ R. Gran ${ }^{\mathrm{r}}$ A. Habig ${ }^{\mathrm{r}}$ Y. Fukuda ${ }^{\mathrm{s}}$

T. Sato ${ }^{\text {s }}$ Y. Itow ${ }^{\mathrm{t}}$ T. Koike ${ }^{\mathrm{t}}$ T. Tanaka ${ }^{\mathrm{t}}$ C.K. Jung ${ }^{\mathrm{u}}$ T. Kato ${ }^{\mathrm{u}}$

K. Kobayashi ${ }^{u}$ C. McGrew ${ }^{u}$ A. Sarrat ${ }^{u}$ R. Terri ${ }^{u}$

C. Yanagisawa ${ }^{\text {u N}}$ N. Tamura ${ }^{\mathrm{V}}$ Y. Idehara ${ }^{\mathrm{w}}$ M. Sakuda ${ }^{\mathrm{w}}$

M. Sugihara ${ }^{w}$ Y. Kuno ${ }^{\mathrm{x}}$ M. Yoshida ${ }^{\mathrm{x}}$ S.B. Kim ${ }^{\mathrm{y}}$ B.S. Yang ${ }^{\mathrm{y}}$

J. Yoo ${ }^{\text {y }}$ T. Ishizuka ${ }^{\text {aa }}$ H. Okazawa ${ }^{z}$ Y. Choi ${ }^{\text {ab }}$ H.K. Seo ${ }^{\text {ab }}$

Y. Gando ${ }^{\text {ac }}$ T. Hasegawa ${ }^{\text {ac }}$ K. Inoue ${ }^{\text {ac }}$ Y. Furuse ${ }^{\text {ad }}$ H. Ishii ${ }^{\text {ad }}$

K. Nishijima ${ }^{\text {ad }}$ H. Ishino ${ }^{\text {ae }}$ Y. Watanabe ${ }^{\text {ae }}$ M. Koshiba ${ }^{\text {af }}$

D. Kielczewska ${ }^{\text {ag }}$ H. Berns ${ }^{\text {ah }}$ K.K. Shiraishi ${ }^{\text {ah }}$ E. Thrane ${ }^{\text {ah }}$ 


\section{K. Washburn ${ }^{\text {ah }}$ R.J. Wilkes ${ }^{\text {ah }}$}

${ }^{a}$ Kamioka Observatory, Institute for Cosmic Ray Research, University of Tokyo, Kamioka, Gifu, 506-1205, Japan

${ }^{\mathrm{b}}$ Research Center for Cosmic Neutrinos, Institute for Cosmic Ray Research, University of Tokyo, Kashiwa, Chiba 277-8582, Japan

${ }^{\mathrm{c}}$ Department of Physics, Boston University, Boston, MA 02215, USA

d Physics Department, Brookhaven National Laboratory, Upton, NY 11973, USA

${ }^{\mathrm{e}}$ Department of Physics and Astronomy, University of California, Irvine, Irvine, CA 92697-4575, USA

${ }^{\mathrm{f}}$ Department of Physics, California State University, Dominguez Hills, Carson, CA 90747, USA

${ }^{\mathrm{g}}$ Department of Physics, Chonnam National University, Kwangju 500-757, Korea

${ }^{\mathrm{h}}$ Department of Physics, Duke University, Durham, NC 27708, USA

${ }^{\mathrm{i}}$ Department of Physics, Gifu University, Gifu, Gifu 501-1193, Japan

${ }^{\mathrm{j} D e p a r t m e n t}$ of Physics and Astronomy, University of Hawaii, Honolulu, HI 96822, USA

${ }^{\mathrm{k}}$ Department of Physics, Indiana University, Bloomington, IN 47405-7105, USA

${ }^{\ell}$ High Energy Accelerator Research Organization (KEK), Tsukuba, Ibaraki 305-0801, Japan

${ }^{\mathrm{m}}$ Department of Physics, Kobe University, Kobe, Hyogo 657-8501, Japan

${ }^{\mathrm{n}}$ Department of Physics, Kyoto University, Kyoto 606-8502, Japan

o Physics Division, P-23, Los Alamos National Laboratory, Los Alamos, NM 87544, USA

${ }^{\mathrm{p}}$ Department of Physics and Astronomy, Louisiana State University, Baton Rouge, LA 70803, USA

${ }^{\mathrm{q}}$ Department of Physics, Massachusetts Institute of Technology, Cambridge, MA 02139, USA

${ }^{\mathrm{r}}$ Department of Physics, University of Minnesota, Duluth, MN 55812-2496, USA

${ }^{\mathrm{s} D e p a r t m e n t}$ of Physics, Miyagi University of Education, Sendai,Miyagi 980-0845, Japan

${ }^{\mathrm{t}}$ Solar Terrestrial Environment Laboratory, Nagoya University, Nagoya, Aichi 464-8602, Japan

${ }^{\mathrm{u}}$ Department of Physics and Astronomy, State University of New York, Stony Brook, NY 11794-3800, USA

'Department of Physics, Niigata University, Niigata, Niigata 950-2181, Japan

${ }^{\mathrm{w}}$ Department of Physics, Okayama University, Okayama 700-8530, Japan

${ }^{x}$ Department of Physics, Osaka University, Toyonaka, Osaka 560-0043, Japan

${ }^{y}$ Department of Physics, Seoul National University, Seoul 151-742, Korea

${ }^{\mathrm{z}}$ International and Cultural Studies, Shizuoka Seika College, Yaizu, Shizuoka, 


\author{
425-8611, Japan

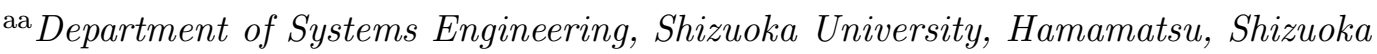 \\ 432-8561, Japan \\ ${ }^{\mathrm{ab}}$ Department of Physics, Sungkyunkwan University, Suwon 440-746, Korea

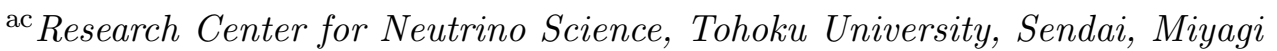 \\ 980-8578, Japan \\ ad Department of Physics, Tokai University, Hiratsuka, Kanagawa 259-1292, \\ Japan

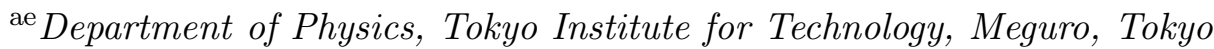 \\ 152-8551, Japan \\ af The University of Tokyo, Tokyo 113-0033, Japan \\ ag Institute of Experimental Physics, Warsaw University, 00-681 Warsaw, Poland

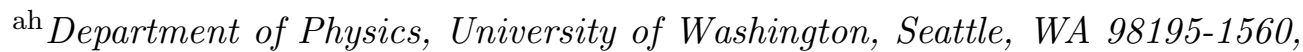 \\ $U S A$
}

\title{
The Super-Kamiokande Collaboration
}

\begin{abstract}
A subset of neutrino-induced upward through-going muons in the Super-Kamiokande detector consists of high energy muons which lose energy through radiative processes such as bremsstrahlung, $e^{+} e^{-}$pair production and photonuclear interactions. These "upward showering muons" comprise an event sample whose mean parent neutrino energy is approximately $1 \mathrm{TeV}$. We show that the zenith angle distribution of upward showering muons is consistent with negligible distortion due to neutrino oscillations, as expected of such a high-energy neutrino sample. We present astronomical searches using these high energy events, such as those from WIMP annihilations in the Sun, Earth and Galactic Center, some suspected point sources, as well as searches for diffuse flux from the interstellar medium.
\end{abstract}

Key words: High-energy neutrinos, Muon energy losses, Astrophysics PACS: 95.55.Ka, 95.55.Vj, 96.40.Pq

\footnotetext{
* Corresponding author. Email: shantanu@neutrino.bu.edu

1 Present address: Department of Physics, Pennsylvania State University, University Park, PA 16802, USA
} 


\section{INTRODUCTION}

In order to select neutrino events with the highest energies, we consider muon neutrino interactions in the rock around the detector, because the effective target volume is very much increased [1]. To separate neutrino-induced muons from cosmic ray muons, we select only upward-going muons, since the background from downward going cosmic ray muons overwhelms any neutrinoinduced muons from above. Muons penetrating the detector have energies of at least several $\mathrm{GeV}$ and point along the neutrino direction within a few degrees, allowing astrophysical studies. Neutrinos originating from cosmic point sources are expected to have harder energy spectra than the background of atmospheric neutrinos. Some of these high energy neutrino-induced muons undergo radiative energy loss. We identify these muons as "showering muons". High energy muons are correlated with high energy neutrinos, which allow us to statistically select an extremely high energy parent neutrino sample from the Super-Kamiokande data. Our aim is to extract this sample for physics and astronomy studies.

The Super-Kamiokande (Super-K) experiment [2] has previously analyzed two topologically distinct categories of upward muons caused by neutrino interactions in the rock below the detector: muons which exit the detector (called "through-going") and those which stop inside the detector (called "stopping") [3]. The parent neutrino energy of upward stopping and throughgoing muons for atmospheric neutrinos is peaked at $\simeq 10 \mathrm{GeV}$ and $\simeq 100$ GeV respectively. In 1646 days of data, Super-Kamiokande detected 1856 upward through-going and 458 upward stopping muons. Oscillation results using only upward through-going muons and stopping muons have been reported in Refs. [3] and [4]. Both upward muon samples have been combined along with the contained events to do the most precise atmospheric neutrino oscillation studies [5,6]. Moreover, astrophysical searches for annihilation signatures of WIMPs [7], point and diffuse sources [8], and neutrinos from GRBs [9] have been done with upward muons.

In this paper we bifurcate the upward through-going muon sample into upward showering and upward non-showering events. This classification is done on the basis of the physical mechanism for muon energy loss above $1 \mathrm{TeV}$. The showering sample is then analyzed to see what these highest-energy events might reveal. 


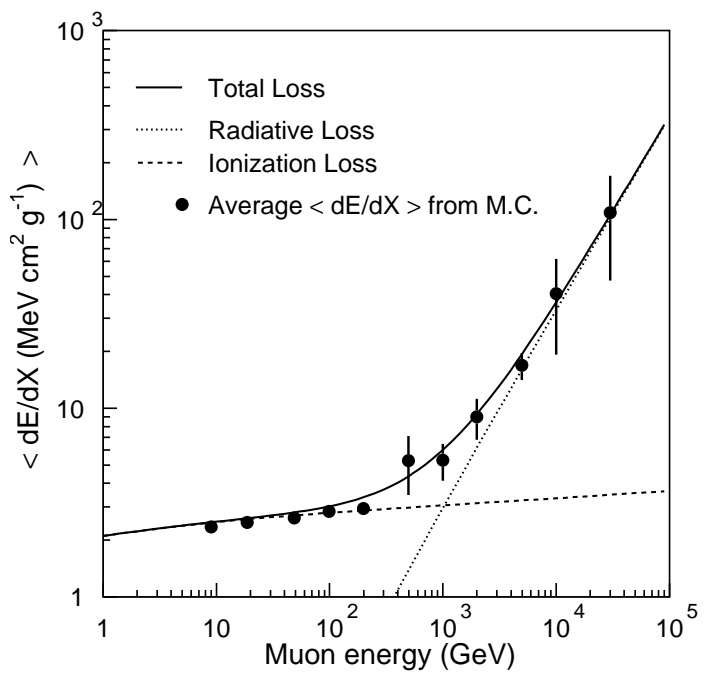

Fig. 1. Average muon energy loss/tracklength for about 100 simulated muons at various energies from $10 \mathrm{GeV}$ to $30 \mathrm{TeV}$. The theoretical curves for energy loss due to ionization and other radiative processes have been obtained from Ref. [11].

\section{MUON ENERGY LOSS}

At low energies, from $100 \mathrm{MeV}$ to $100 \mathrm{GeV}$, muon energy loss is dominated by atomic ionization as described by Bethe-Bloch; the minimum ionization energy loss in water is $1.99 \mathrm{MeV} / \mathrm{cm}$ [10]. At higher energies, radiative processes such as $e^{+} e^{-}$pair production, bremsstrahlung, and photonuclear interactions become important, resulting in catastrophic energy losses with large fluctuations. In water, the critical energy where ionization energy loss is equal to the average radiative energy loss is $1.03 \mathrm{TeV}$ [11]. The relevant processes have recently been reviewed in Refs. [12,13].

To develop an algorithm for showering/non-showering separation and test its efficiency, we used a GEANT 3 based detector simulation to simulate muons at several energies between $10 \mathrm{GeV}$ and $30 \mathrm{TeV}$. We checked this Monte Carlo sample to make sure that the muon energy loss in our detector simulation agrees with calculated values[11]. The average energy loss/tracklength at each muon energy is shown in Fig. 1, in good agreement with the calculation.

All details of the muon energy loss calculations for different processes in GEANT 3 are discussed in Ref. [14]. The cross-section for bremsstrahlung is obtained from Ref. [15]. The energy loss for direct electron-positron pair production is obtained from Ref. [16]. Cross-sections for photonuclear interactions are obtained from Ref. [17]. 


\section{DATA AND MONTE CARLO USED}

For this paper, we used all upward through-going muons from the full SuperK-I dataset from May 1996 to July 2001 spanning 1645.9 days of data. We require that reconstructed tracks have a minimum length of $7 \mathrm{~m}$, which reduces the contamination from photo-produced upward pions to $0.01 \%[18,19]$. This path-length cut of $7 \mathrm{~m}$ corresponds to a muon energy threshold of 1.6 $\mathrm{GeV}$. As discussed in Ref. [5], all upward muon events require between 8,000 and 1,750,000 photoelectrons. A specialized analysis looking for upward muons in events with greater than 1,750,000 photoelectrons has been reported elsewhere [20].

We applied the showering algorithm to simulated upward through-going muons from the standard Super-Kamiokande atmospheric neutrino Monte Carlo sample [5]. The input atmospheric neutrino flux used for this Monte Carlo sample was from Ref. [21] for neutrino energies up to $1 \mathrm{TeV}$. At $1 \mathrm{TeV}$, the calculated flux in Ref. [22] was rescaled to that in Ref. [21], and used for neutrino energies up to $100 \mathrm{TeV}$. The uncertainty in the absolute atmospheric neutrino flux is about $20 \%$ below $1 \mathrm{TeV}$ and and ranges from $25 \%$ [23] to $40 \%$ [24,25] around $1 \mathrm{TeV}$. The atmospheric neutrino flux in Ref. [23] is about 15-20\% greater at $1 \mathrm{TeV}$ as compared to Ref. [21]. Neutrino interactions with the rock outside the detector (assumed to be "standard rock") as well as in the water were simulated using the NEUT [26] interaction package. Neutrino interactions in the rock were simulated up to $4 \mathrm{~km}$ from the center of the detector. The total livetime simulated was equal to 100 years.

\section{LIGHT PRODUCTION MODEL}

Below the critical energy, an ionizing muon produces a constant amount of Cherenkov light per unit tracklength. However, a muon which undergoes radiative energy loss produces high-energy photons which create electron-positron pairs, thus increasing the total Cherenkov light in the detector. If we can calibrate the total Cherenkov light emitted by a normally ionizing muon (after accounting for the various sources of light attenuation in Super-K) then any electromagnetic shower associated with the muon will emit excess light over this amount. Thus we need to calibrate the total "luminosity" of ionizing muons with energy well below the critical energy of $1 \mathrm{TeV}$.

Using the muon entry point and direction as inputs, and accounting for the effective water attenuation length $\left(L_{a t t}\right)$ and geometrical acceptance corrections, we apply various corrections to the raw PMT photoelectrons. The corrected 
number of photoelectrons of each PMT in the Cherenkov cone is:

$$
q_{\text {corr }}(\text { corr. pe })=K \frac{q_{\text {raw }} d_{w} e^{\left(\frac{d_{w}}{L_{a t t}}\right)}}{F(\theta)}
$$

where $q_{\text {raw }}$ is the raw number of photoelectrons detected by each PMT; $d_{w}$ is the distance traveled by the photons from the point along the muon track where the photon is emitted to the PMT which detects it; $F(\theta)$ accounts for the PMT angular acceptance and shadowing; and $K$ is a normalization constant $\left(=1 / 2500 \mathrm{~cm}^{-1}\right)$ which makes the corrected photoelectrons the same order of magnitude as the original raw photoelectrons. With these corrections to the charge of each PMT, the units of $q_{\text {corr }}$ and other terms obtained from $q_{\text {corr }}$ are corrected photoelectrons.

We then calculate the average charge in small tracklength intervals $(50 \mathrm{~cm})$ along the muon track:

$$
Q_{\text {corr }}^{i}=\frac{1}{N_{p m t}} \sum_{k=1}^{N_{p m t}} q_{c o r r},
$$

where $N_{p m t}$ is the number of PMTs whose projected distance along the muon track is within the $50 \mathrm{~cm}$ path-length interval. The statistical uncertainty in $Q_{\text {corr }}^{i}$ is given by:

$$
\sigma_{Q_{\text {corr }}^{i}}^{2}=\frac{1}{N_{p m t}^{2}} \sum_{k=1}^{N_{p m t}} \frac{\left(q_{\text {corr }}\right)^{2}}{q_{\text {raw }}},
$$

assuming that the error in raw PMT charge arises from the square-root of the number of photoelectrons. We then plot $Q_{\text {corr }}^{i}$ along the muon track. An example plot for a typical ionizing Monte Carlo muon event can be seen in the top panel of Fig. 2. We also calculate the average of $Q_{c o r r}^{i}$ along the muon track as follows:

$$
\left\langle Q_{\text {corr }}\right\rangle=\frac{\sum_{i=4}^{N-3}\left(Q_{\text {corr }}^{i} / \sigma_{Q_{\text {corr }}^{i}}^{2}\right)}{\sum_{i=4}^{N-3} 1 / \sigma_{Q_{\text {corr }}^{i}}^{2}},
$$

where $N$ indicates the number of $50 \mathrm{~cm}$ bins in which the muon track is subdivided. In Eqn. 4, the sum goes from 4 to $N-3$, since the first and last $1.5 \mathrm{~m}$ from the muon track are excluded. This is because corrections for PMT acceptance and shadowing are not accurately modeled near the wall.

In addition, because of the effects of light scattering into the Cherenkov cone, longer path-length muons have greater corrected mean charge compared to short path-length muons. This is accounted for by using a path-length correction to the average charge of an ionizing muon. The corrected charge distribution of a typical ionizing muon is compared to that of a showering muon with the same entry point and direction in the bottom panel of Fig. 2. Our aim is to construct a function which quantitatively distinguishes between these based 

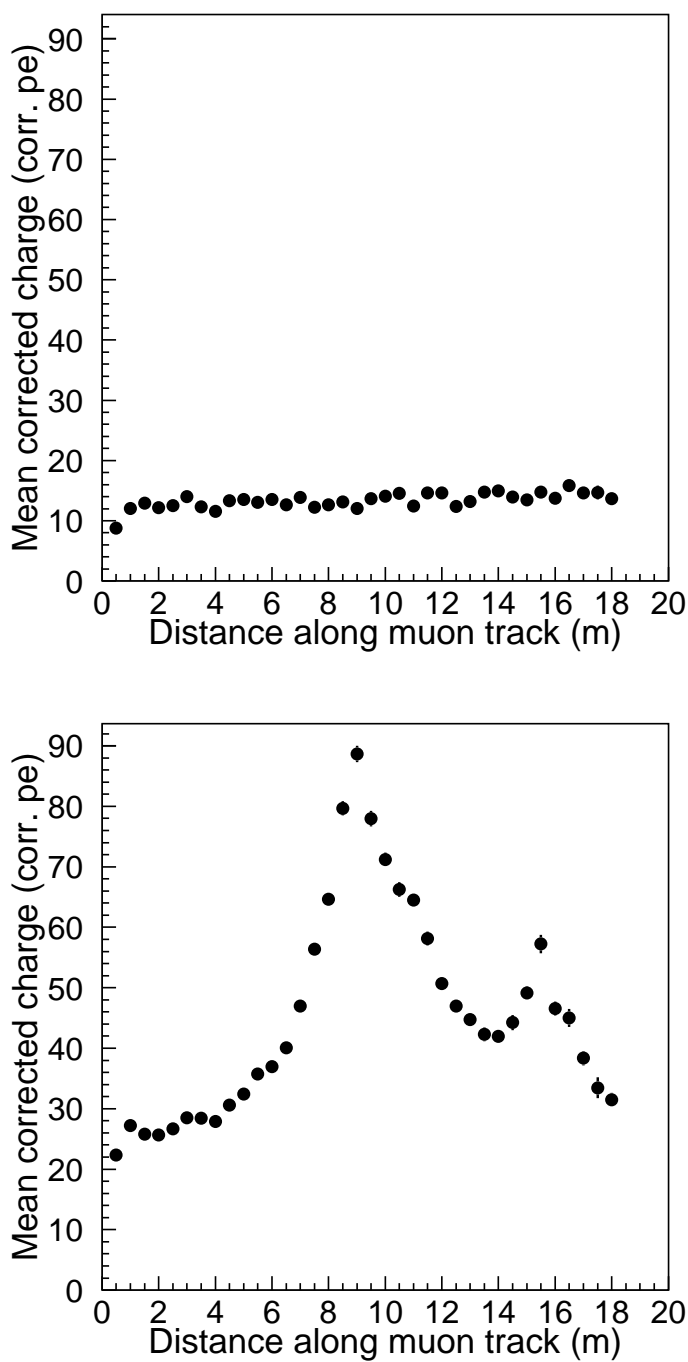

Fig. 2. The corrected photoelectrons for an example simulated ionizing muon with energy $20 \mathrm{GeV}$ (top) and a showering muon with energy $10 \mathrm{TeV}$ (bottom) simulated with the same entry point and direction.

on shape and total collected photoelectrons.

\section{SELECTION ALGORITHM FOR SHOWERING MUONS}

We define a showering $\chi^{2}$ as follows:

$$
\chi_{\text {showering }}^{2}=\frac{1}{N-6} \sum_{i=4}^{N-3}\left\{\frac{\left[Q_{\text {corr }}^{i}-\left\langle Q_{\text {corr }}\right\rangle\right]}{\sigma_{Q_{\text {corr }}^{i}}}\right\}^{2},
$$

where $N$ is same as in Eqn. 4; $\left\langle Q_{\text {corr }}\right\rangle$ is defined in Eqn. $4 ; \sigma_{Q_{\text {corr }}^{i}}$ is the statistical error in $Q_{\text {corr }}^{i}$ and defined in Eqn. 3. Equation 5 measures deviations 
in the histogram from a flat distribution.

About $0.5 \%$ of events in the Monte Carlo consist of through-going muons with bad fits or stopping muons which are misclassified as through-going muons. These events show large values of $\chi^{2}$ even though the total number of photoelectrons in such events is very small. To eliminate these cases, we define another variable to distinguish between a showering and non-showering muon:

$$
\Delta=\left[\left\langle Q_{\text {corr }}\right\rangle-Q_{\text {exp }}(l)\right]
$$

where $\left\langle Q_{\text {corr }}\right\rangle$ is defined in Eqn. 4 and $Q_{\text {exp }}(l)$ is a path-length dependent estimate of the expected charge of a normally ionizing muon. The magnitude of $\Delta$ indicates how much more Cherenkov light is present relative to a normally ionizing muon. The comparison of these variables for data and 100 year atmospheric neutrino Monte Carlo is shown in Fig. 3. The distributions of these variables for data and Monte Carlo is in good agreement.

We applied this algorithm to the 37287 upward through-going muon events in the 100 year neutrino Monte Carlo [5]. The scatter plots of the distribution of $\chi_{\text {showering }}^{2}$ and $\Delta$ are shown in Fig. 4 for a 10 year subset of the 100 year Monte Carlo. They are presented separately for samples of "true" showering and non-showering muons, which are defined based on the true muon energy loss per unit tracklength $\Delta E / L$ (where $\Delta E / L$ is the true muon energy loss in the inner detector for a muon of path-length $L$ ), with the separation value equal to $2.85 \mathrm{MeV} / \mathrm{cm}$.

The separation between showering and non-showering events is decided by the line shown in Figure. 4. With this cut we found a total of 5747 upward showering events in the Monte Carlo. We then calculate the efficiency, defining true showering muons as those with $\Delta E / L>2.85 \mathrm{MeV} / \mathrm{cm}$. This cut selects about $70 \%$ of such events, while the misidentification of events with true $\Delta E / L<2.85 \mathrm{MeV} / \mathrm{cm}$ is about $5 \%$. These cuts provide reasonable purity and background contamination in forming a sample for astrophysical studies and neutrino oscillation.

The parent neutrino energy spectra of all upward stopping, non-showering through-going, and showering through-going muons are shown in Fig. 5. The mean parent neutrino energy of upward showering muon events is peaked at 1 $\mathrm{TeV}$. Thus the showering dataset constitutes the highest energy neutrinos seen in Super-K. The total number of upward showering muons, is approximately one-fifth the total number of upward through-going muons.

The estimated muon angular resolution of upward showering muon events is about $1.4^{\circ}$, where angular resolution is defined as the average angular separation between true muon direction and reconstructed muon direction. The corresponding angular resolution for upward stopping and through-going muons 

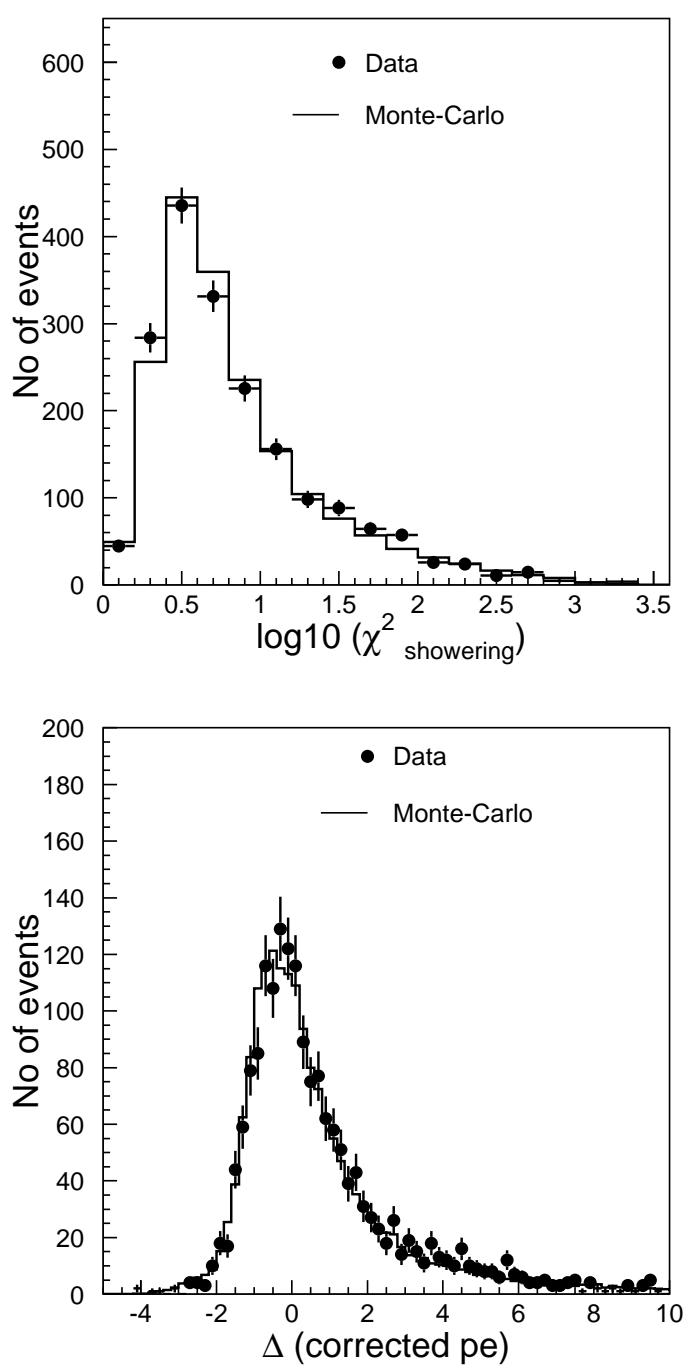

Fig. 3. Comparison of showering variables as described in Eqn. 5 and 6 for Monte Carlo and 1645.9 days upward through-going muon data. The solid line is normalized to total number of events.

is about $2.4^{\circ}$ and $1.3^{\circ}$ respectively. For higher energy muons, the additional photons generated through radiative processes make it harder to reconstruct the muon track direction. Hence the angular resolution is slightly worse for showering muons compared to the non-showering muons.

\section{BACKGROUND SUBTRACTION}

When we applied the showering muon algorithm to the upward through-going muon sample from the 1646 days of data, we found a total of 318 showering events. The upward muon data sample is contaminated near the horizon from downward going cosmic ray muons which appear as upward going because of 

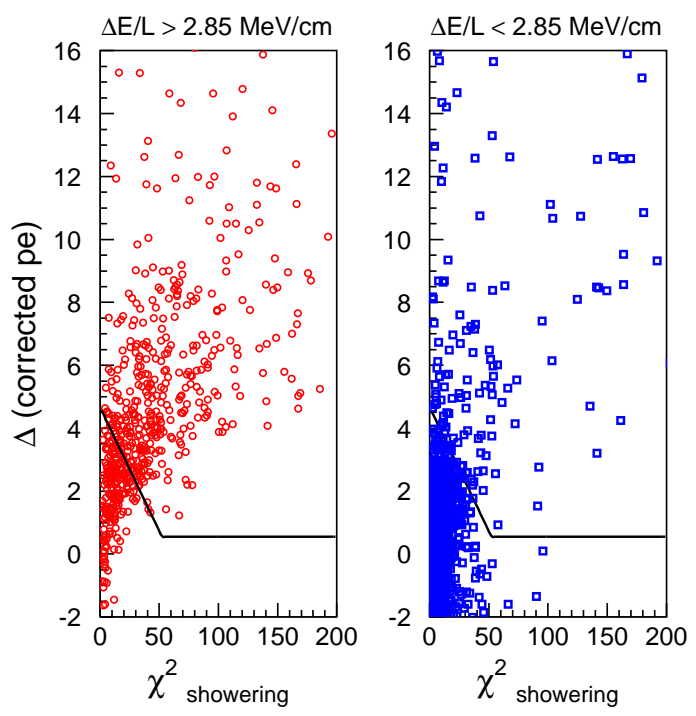

Fig. 4. Distribution of $\Delta$ vs $\chi_{\text {showering }}^{2}$ for events from a 10 year Monte Carlo sample (which is a subset of the 100 year atmospheric neutrino Monte Carlo) with true $\Delta E / L>2.85 \mathrm{MeV} / \mathrm{cm}$ (left panel), i.e. "true showering events," and true $\Delta E / L<$ $2.85 \mathrm{MeV} / \mathrm{cm}$ (right panel), i.e. "true non-showering events." The solid line indicates the cut used to separate showering from non-showering muons.

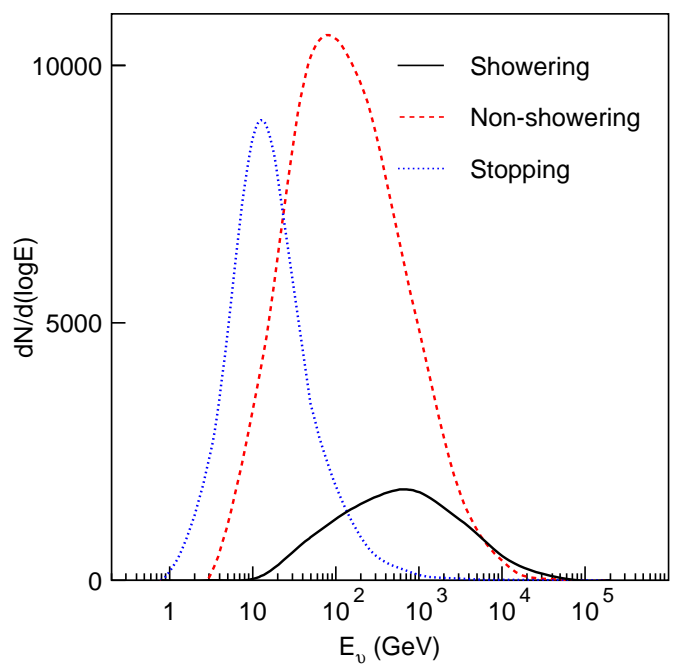

Fig. 5. Neutrino energy spectra of all 3 categories of upward muons in Super-Kamiokande in 100 yrs of atmospheric neutrino Monte Carlo.

multiple Coulomb scattering and the finite angular resolution of the fitters. To estimate this background in the showering muon sample, we use the same procedure as that applied for stopping and through-going muons [5]. We apply the showering algorithm and select a subsample of near horizon downward through-going muons with $0<\cos \Theta<0.08$, where $\Theta$ is the zenith angle defined with the vertical upward direction at $\cos \Theta=-1$. We then estimate the background from the downward muon sample by extrapolation below the horizon [5]. The zenith and azimuthal distribution of near-horizontal shower- 

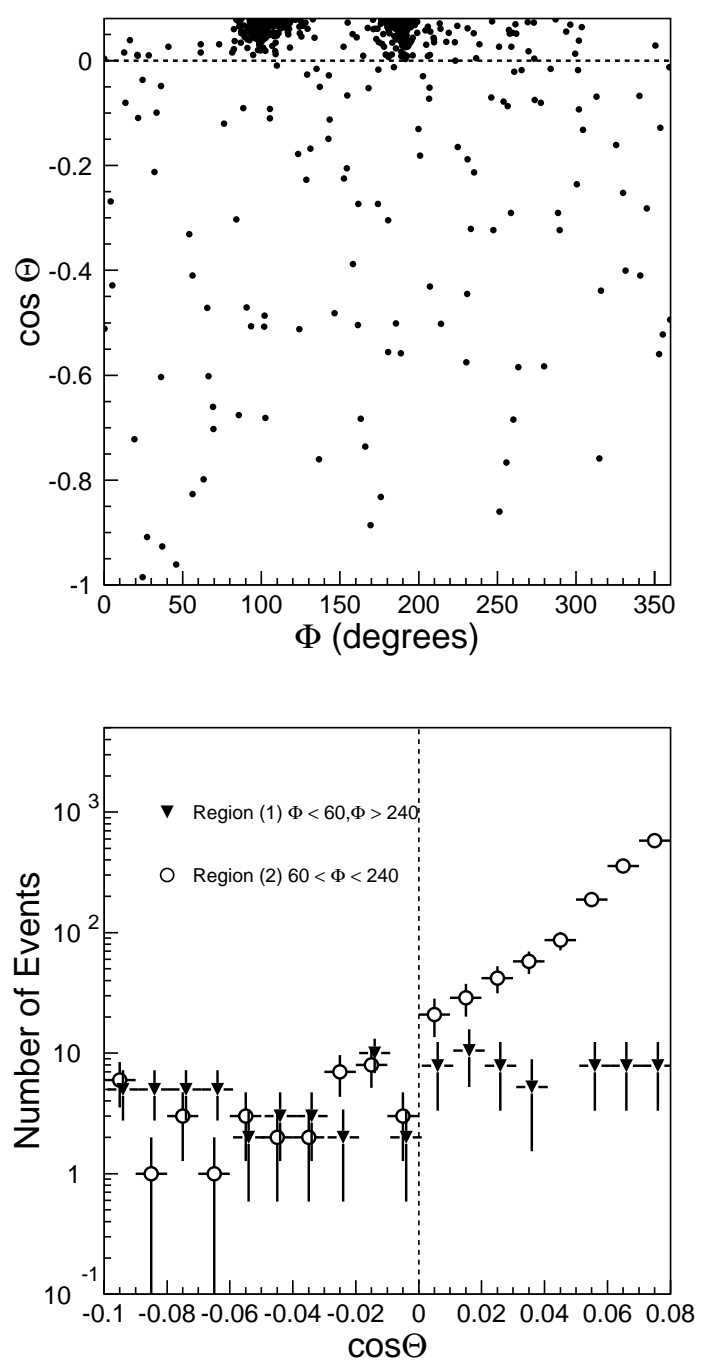

Fig. 6. Zenith and azimuthal distribution of showering muons in the region of $-1.0<\cos \Theta<0.08$. Regions (1) and (2) in bottom panel refer to thick and thin parts of the mountain respectively.

ing muon events is shown in Fig. 6. The expected background from showering muons is estimated to be $9.15_{-5.3}^{+13.0}$, all in the bin from $0<\cos \Theta<-0.1$. This background is subtracted from the upward showering muon dataset for oscillation studies. For astrophysical studies, we assign a weight to this zenith angle bin which is equal to the ratio of background-subtracted to non-background subtracted events.

\section{OSCILLATION ANALYSIS}

Previous analyses have shown that the Super-K atmospheric neutrino dataset is consistent with neutrino oscillations with $\Delta m^{2} \simeq 0.0025 \mathrm{eV}^{2}$ and $\sin ^{2} 2 \theta=$ 


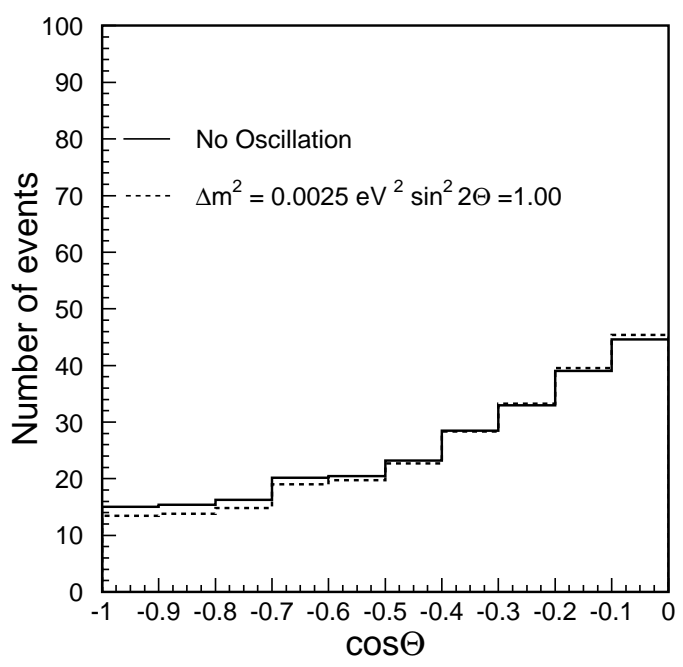

Fig. 7. The expected zenith angle distribution of upward showering events with and without neutrino oscillations. Both histograms are normalized to the livetime of the dataset.

$1.0[5,27]$. Given these neutrino oscillation parameters, the oscillation probability is negligible for neutrinos with an energy of $1 \mathrm{TeV}$ at all path-lengths through the Earth. The zenith angle distributions of upward showering muons without oscillation and with oscillated parameters obtained from Ref. [5] are shown in Fig. 7. As expected, the difference between these distributions is negligible. We will demonstrate this by comparing the zenith angle distribution of data and Monte Carlo.

The chi-square function used for comparison of data and Monte Carlo is the same as that used for our published oscillation analysis [5], which is based on the pull method [28]:

$$
\begin{aligned}
\chi^{2} & =\sum_{i=1}^{10}\left[\frac{N_{i}^{\text {obs }}-N_{i}^{\text {exp }}}{\sigma_{i}^{\text {stat }}}\right]^{2}+\sum_{j=2}^{N_{\text {sys }}}\left[\frac{\epsilon_{j}}{\sigma_{j}^{\text {sys }}}\right]^{2} \\
N_{i}^{\text {exp }} & =N_{i}^{\text {osc }}\left(1+\sum_{j=1}^{N_{\text {sys }}} f_{i}^{j} \epsilon_{j}\right)
\end{aligned}
$$

In Eqn. 7, $N_{i}^{\text {obs }}$ is the number of observed events in $i^{t h}$ bin, $N_{i}^{e x p}$ is the expected number of events considering both oscillation and systematic uncertainties, $\sigma_{\text {stat }}^{i}$ combines statistical uncertainties in the data and Monte Carlo simulation, $N_{\text {sys }}$ is the number of systematic errors used for the fit, and $N_{i}^{\text {osc }}$ is the expected number of events in this bin assuming $\nu_{\mu}$ to $\nu_{\tau}$ oscillation without considering the contribution from systematic uncertainties. The factor $f_{j}^{i}$ represents the fractional change in the predicted event rate in the $i^{\text {th }}$ bin due to a variation of the parameter $\epsilon_{j}$. The second sum in the $\chi^{2}$ definition collects 
Table 1

Summary of systematic errors and the best fit at null oscillation used for upward showering muons.

\begin{tabular}{|c|c|c|c|}
\hline No & Systematic uncertainty & $\sigma(\%)$ & Best-fit $(\%)$ \\
\hline 1 & Absolute Normalization & Free & 20.4 \\
2 & $K / \pi$ ratio & 20 & -6.75 \\
3 & Axial vector Mass $\left(M_{A}\right)$ & 10 & 0.15 \\
4 & Multi-pion production (model-dependence) & 1 & -0.44 \\
\hline
\end{tabular}

the contributions from the systematic uncertainties in the expected neutrino rates.

As we are doing oscillation analysis with only one data sample, and since the absolute normalization is considered to be free as done in Ref. [5], we only need to consider those systematic error coefficients for which the response is non-uniform in different zenith angle bins. The effect of all systematic terms for which $f_{i}^{j}$ is same for all $i$ can be incorporated in the absolute normalization. Of all the systematic terms used in Ref. [5], there are only three for which the response is different in various zenith angle bins. These are shown in Table 1.

The atmospheric neutrino Monte Carlo does not include the attenuation of neutrino flux in the Earth. We did an estimate of the effect of neutrino absorption for the showering muons using the models in Ref. [29] and the expected decrease in number of events is about $1.1 \%$. Therefore we do not incorporate it into the zenith angle prediction. Hence $N_{\text {sys }}=4$ in Eqns. 7 and 8 .

We vary these four $\epsilon_{j}$ in order to minimize $\chi^{2}$ for each choice of oscillation parameters $\sin ^{2} 2 \theta$ and $\Delta m^{2}$. Among these, only three contribute to the $\chi^{2}$ because there are no constraints on the absolute normalization. The minimum $\chi^{2}$ value, $\chi_{\min }^{2}=3.54 / 7$ DOF, is located at $\left(\sin ^{2} 2 \theta=1.0, \Delta m^{2}=\right.$ $\left.1.05 \times 10^{-2} \mathrm{eV}^{2}\right)$. The number of DOF is found by 10 terms in the $\chi^{2}$ sum plus three systematic constraints in the $\chi^{2}$ sum minus four minimized parameters minus the two physics parameters of $\sin ^{2} 2 \theta$ and $\Delta m^{2}$. For null oscillation, $\left(\sin ^{2} 2 \theta=0\right)$, we found a $\chi^{2}$ value of 6.21 for $9 \mathrm{DOF}$, where only the overall normalization is a free parameter. The best fit values for the systematic uncertainties for null oscillations are indicated in Table 1. In Eqn. 8, the best-fit value of $\epsilon$ for absolute normalization for the case of null oscillation is about $20.4 \%$. The minimum $\chi^{2}$ is not significantly different than the $\chi^{2}$ obtained for null oscillation.

Thus, we satisfy an important consistency check of the neutrino oscillation parameters determined in Ref. [5], namely, that this dataset (with mean neutrino energy of $\simeq 1 \mathrm{TeV}$ ) is consistent with null oscillation. In future studies, 


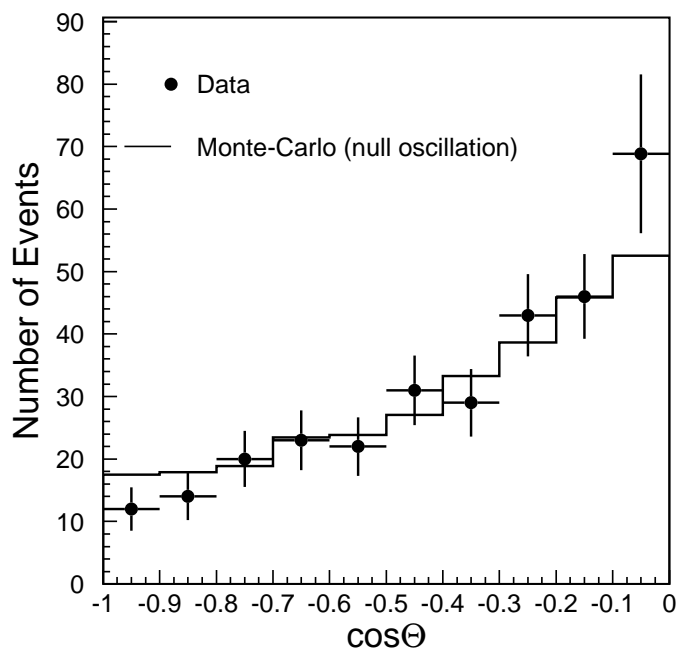

Fig. 8. Observed number of upward showering muons as a function of zenith angle. The dots represent the observed events with statistical error bars. The solid line shows the expectation at null oscillation evaluated using Eqn. 8 and from best fit values in Table 1.

this dataset could serve as an additional high energy bin, constraining high $\Delta m^{2}$ solutions as well as certain non-standard oscillation scenarios [30,31]. The zenith angle distributions of upward showering muon data along with the expected distribution from null oscillations and using the best fit values from Table 1 are shown in Fig. 8.

\section{ASTROPHYSICAL SEARCHES}

The high energy nature of this subset of the Super-K neutrino data provides an advantage when searching for astrophysical neutrino point sources, neutrinos from WIMP annihilation, and diffuse neutrino flux from the galactic plane. The main advantage in using this high energy sample for astronomy is that the atmospheric neutrino background is very much reduced due to the steeply falling atmospheric neutrino spectrum [32,33,34,35]. Furthermore, the high momentum of the incoming neutrino results in a muon which points more closely to the initial neutrino direction at higher energies. The mean angular separation between an upward showering muon and its parent neutrino is $2.1^{\circ}$ (assuming an atmospheric neutrino spectrum.) The corresponding numbers for upward through-going muons and upward stopping muons are approximately $2.9^{\circ}$ and $8.7^{\circ}$ respectively. We also estimated the mean angular direction between upward showering muons and their parent neutrinos for different parent neutrino energy spectra. For cosmic ray spectra and $1 / E^{2}$ spectra, the mean angular separation is $1.8^{\circ}$ and $1.7^{\circ}$ respectively. Therefore, we shall redo the searches for steady state point and diffuse astrophysical sources from Ref. [8] 
with the showering muon dataset. There is no additional advantage in using this dataset for transient astrophysical searches, since the timing window coincidence already provides a strong cut to reduce the background.

\subsection{WIMP searches}

We have performed searches for WIMP annihilations in the center of the Earth, Sun and Galactic Center using upward through-going muons [7]. Here we repeat the same search using upward showering muons. The cone size which contains most of the WIMP signal is inversely proportional to the WIMP mass. Since only high-mass WIMPs produce upward showering muons, we perform these searches in cones only up to $5^{\circ}$. Such a cone contains $90 \%$ of the signal for a WIMP of mass $1438 \mathrm{GeV}$ from the Earth and $1000 \mathrm{GeV}$ from the Sun and Galactic Center [7]. The observed data and expected background (evaluated in the same way as in Ref. [7]) are shown in Table 2. Since, there is no statistically significant excess in any of the search cones, we do not see any evidence for WIMP-induced upward showering muons in our dataset.

Because of the reduced background from atmospheric neutrinos in this data sample, we expect to obtain better flux limits with this sample, compared to those in Ref. [7]. These flux limits calculated using only upward showering muons are plotted for the Earth, Sun and Galactic Center in Fig. 9, 10, and 11, respectively. The cutoff WIMP mass used for the calculation of WIMP flux limits using only the showering muon dataset is $1500 \mathrm{GeV}$ for the Earth and the Galactic Center, and $2000 \mathrm{GeV}$ for the Sun. This cutoff mass was calculated by determining the minimum mass for which the neutrino energy spectrum from WIMP annihilation is peaked at $1 \mathrm{TeV}$. This spectrum was calculated by assuming the same branching ratio in all the available annihilation channels for a given WIMP mass, and by using the analytic expressions for neutrino energy spectra calculated in Ref. [36]. The reason for the slightly higher cutoff for the Sun is because, unlike the Earth [37] and Galactic Center [38], energetic neutrinos lose energy or get absorbed by neutral and charged currents in the Sun $[36,39,40,41,42]$. To evaluate the WIMP flux limits with showering muons, we evaluated the showering efficiency at different WIMP masses. Note that we have also extended the flux limits with all upward muons down to $10 \mathrm{GeV}$ (from $18 \mathrm{GeV}$ in Ref. [7]) by including the contributions of upward stopping muons. We have also shown for comparison the corresponding limits from AMANDA-II and Baikal for the Earth [43,44], AMANDA-II limits for the Sun [45], and MACRO limits for the Galactic Center [46]. The WIMP flux limits from all other detectors can be found in Ref. [7]. 


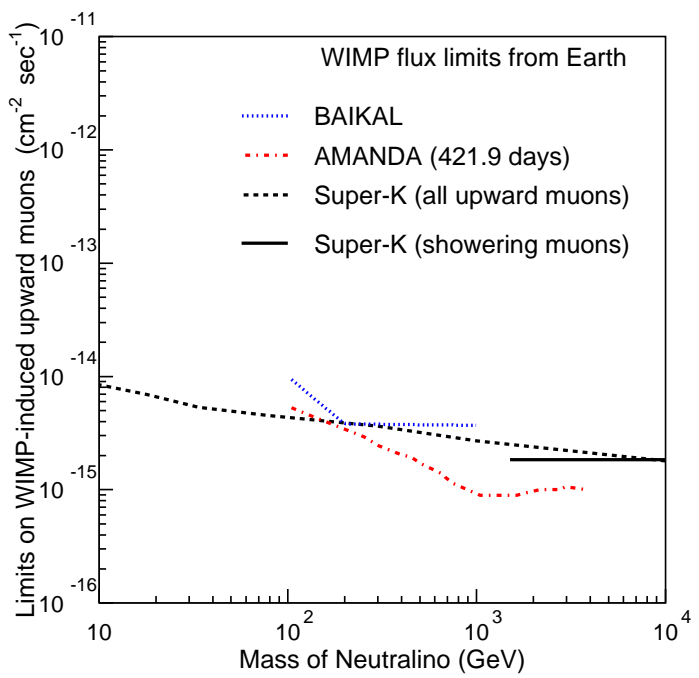

Fig. 9. Super-K WIMP-induced flux limits of upward showering muons and all upward muons from the Earth as a function of mass.

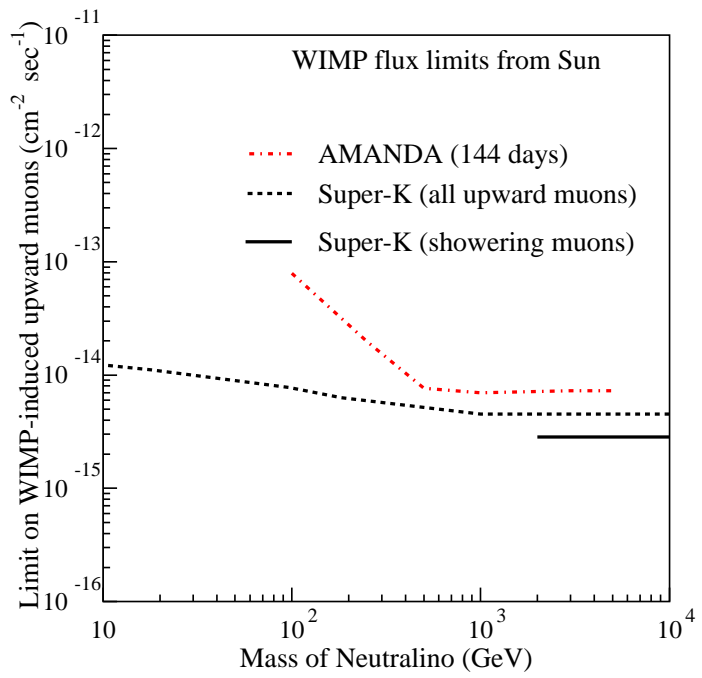

Fig. 10. Super-K WIMP-induced flux limits of upward showering muons and all upward muons from the Sun as a function of mass.

\subsection{Search for a signal from potential point sources}

The equatorial coordinate distribution of upward showering muons is shown in Fig. 12. We look for signatures of neutrinos from 62 suspected point sources. Fifty-two of these sources were analyzed previously in Refs. [8,46] and many of these satisfy some of the features of the "beam dump model" [47,48]. Most of these sources are either supernova remnants, pulsars, magnetars or different types of active galactic nuclei. Some of these have been detected in $\mathrm{TeV} \gamma$ rays [49]. We have also considered some additional promising neutrino point 


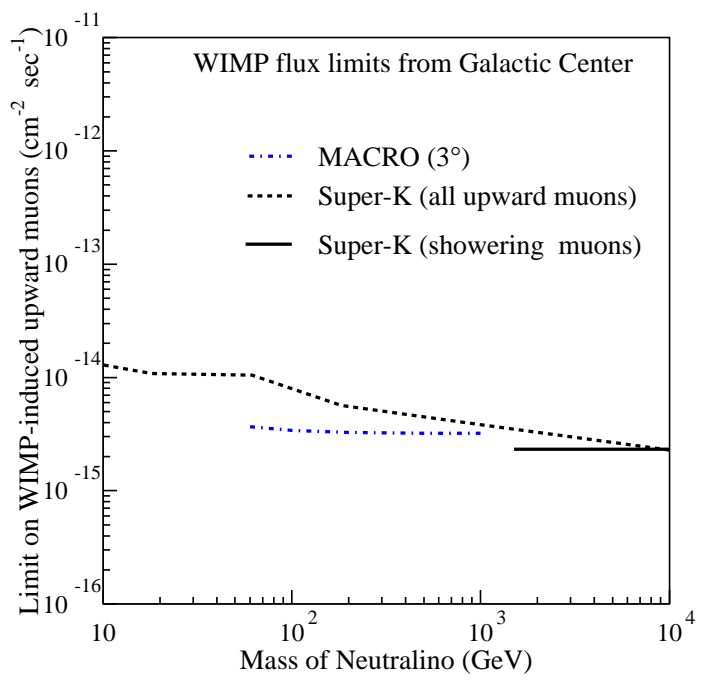

Fig. 11. Super-K WIMP-induced flux limits of upward showering muons and all upward muons from the Galactic Center as a function of mass.

Table 2

Observed and expected upward showering muons in cones with half-angles $3^{\circ}$ and $5^{\circ}$ around the Earth, Sun and Galactic Center.

\begin{tabular}{|c|cc|cc|cc|}
\hline & \multicolumn{2}{|c|}{ Earth } & \multicolumn{2}{c|}{ Sun } & \multicolumn{2}{c|}{ Galactic Center } \\
\hline Cone & Data & Background & Data & Background & Data & Background \\
\hline $3^{\circ}$ & 0 & 0.1 & 0 & 0.2 & 0 & 0.4 \\
\hline $5^{\circ}$ & 0 & 0.5 & 0 & 0.5 & 0 & 0.8 \\
\hline
\end{tabular}

sources following the recent results from H.E.S.S $\gamma$-ray telescope $[51,52]$.

In this search, we look for a statistically significant excess in a cone of angular size $3^{\circ}$ around these sources. Such a cone size would contain $90 \%$ of the signal for a $1 / E^{2}$ neutrino spectra. The background was evaluated using atmospheric neutrino Monte Carlo. Each Monte Carlo event was assigned a time sampled from the upward showering muon distribution, in order to match the livetime distribution of the observed events. The observed data and expected background are shown in Table 3 . As we can see, there is no statistically significant excess in any of these cones. For each source, we calculate the muon flux limit at $90 \%$ c.l. using the method described in Ref. [50]. To calculate the neutrino flux limits, we assumed a $E^{-2}$ neutrino energy spectrum. This spectrum arises in a number of astrophysical circumstances such as beam dumps and shock acceleration. The calculation of neutrino flux limits from the showering muon flux limits is as follows.

The total flux of upward muons above an energy threshold $E_{\mu}^{\min }$ for a $1 / E^{2}$ 
spectra is given by [47]:

$$
\Phi_{\mu}=A \int_{E_{\mu}^{\min }}^{\infty} d E_{\nu} P_{\mu}\left(E_{\nu}, E_{\mu}^{\min }\right) E_{\nu}^{-2}
$$

In Eqn. 9, $A$ is a normalization constant for the differential neutrino flux, $P_{\mu}\left(E_{\nu}, E_{\mu}^{\min }\right)$ is the probability that a neutrino with energy $E_{\nu}$ produces a muon with energy greater than $E_{\mu}^{\min }$. The total flux of upward showering muons $\left(\Phi_{\mu}^{\text {showering }}\right)$ is then given by :

$$
\Phi_{\mu}^{\text {showering }}=k_{\text {cone }} k_{\text {algo }} \int_{0}^{\infty} d E_{\mu}^{\min } \frac{d \Phi_{\mu}\left(\geq E_{\mu}^{\min }\right)}{d E_{\mu}^{\min }} \epsilon\left(E_{\mu}^{\min }\right)
$$

In Eqn. 10, $\epsilon\left(E_{\mu}^{\min }\right)$ is the probability for a muon to undergo radiative energy losses as a function of muon energy, $k_{\text {algo }}$ is the efficiency of our algorithm to detect a true showering muon using the cuts in Sect. 5 , and $k_{\text {cone }}$ is the fraction of the signal which falls within the $3^{\circ}$ cone. For each source, we solve for $A$ by substituting the obtained showering muon flux limits in the left-hand-side of Eqn. 10 and using $\Phi_{\mu}$ as evaluated from Eqn. 9.

To evaluate the above integrals, we calculated the values of $P_{\mu}\left(E_{\nu}, E_{\mu}^{\min }\right)$ using codes provided by M. Reno [29] (2005, private communication). More details on the assumptions used for this calculation can be found in Ref. [20]. The efficiency $\epsilon\left(E_{\mu}^{\min }\right)$ was calculated by first finding the fraction of muons from the mono-energetic Monte Carlo (used in Fig. 1) with $d E / d X>2.85$ $\mathrm{MeV} / \mathrm{cm}$ at different muon energies, after which we applied a curve fit. The values of $k_{\text {algo }}$ and $k_{\text {cone }}$ were estimated for a $1 / E^{2}$ spectra and each of them is equal to 0.9. Evaluating the integrals in Eqn. 10, the value of $A$ for each source is given by $A=3.52 \times 10^{8} \Phi_{\mu}^{\text {showering }} \mathrm{GeV}^{-1}$. Once $A$ is determined, the integrated neutrino flux limits were obtained by evaluating $A \int_{E_{\nu}^{\min }}^{\infty} E^{-2} d E$, where $E_{\nu}^{\text {min }}$ is the minimum neutrino energy which would make a showering muon and is chosen to be $10 \mathrm{GeV}$. This gives us $90 \%$ c.l. neutrino flux limits for a given source from the corresponding $90 \%$ c.l. showering muon flux limits. These muon and neutrino flux limits for all the sources are shown in Table 3. The corresponding neutrino flux limits for some of these sources from MACRO and AMANDA-II can be found in Refs [46] and Refs. [53,54]. A plot showing a comparison of the neutrino flux limits from these detectors for some sample sources as a function of declination can be found in Fig. 13. 


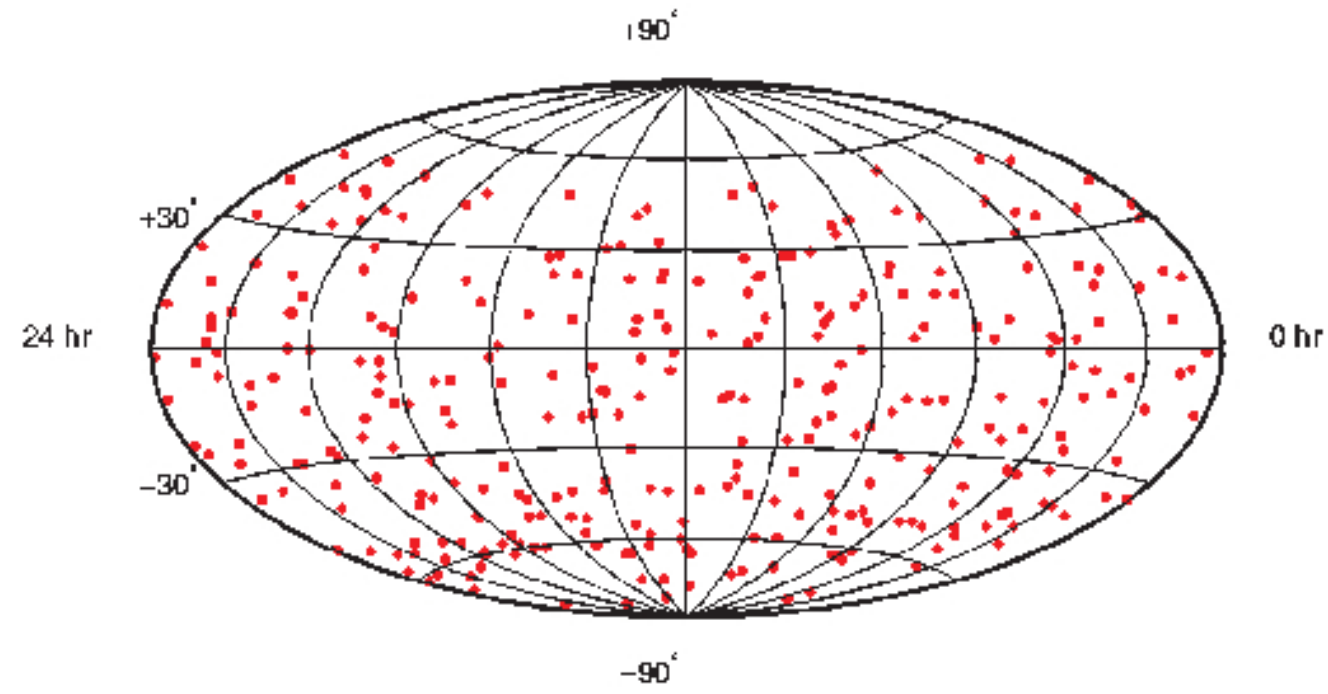

Fig. 12. Equatorial coordinate distribution of all upward showering muons.

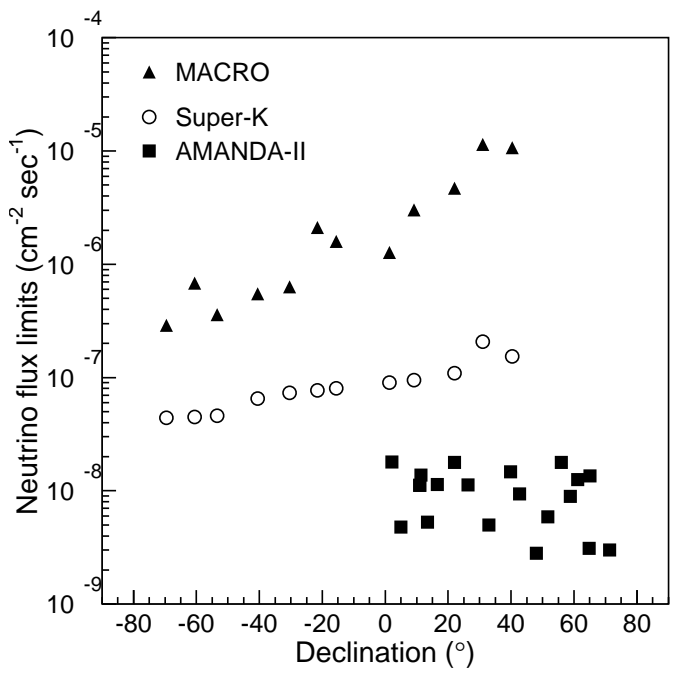

Fig. 13. Neutrino flux limits from Super-K, AMANDA-II [54] (for $E^{-2}$ neutrino spectra) and MACRO [46](for $E^{-2.1}$ neutrino spectra) as a function of declination for some selected sources.

\subsection{Search for unexpected point sources}

In order to search for a signal from an unexpected point source (which may not have any electromagnetic signature), we have looked for an excess of events within a cone of half-angle $4^{\circ}$ around any upward showering muon event. The background is estimated using the atmospheric $\nu$ Monte Carlo in the same way as in Sect. 6.1. The distribution of the number of observed events with the $4^{\circ}$ cone is fit well by a Poisson distribution with a mean of 0.35 events. The comparison of data and Monte Carlo as well as results from the Poisson fit is shown in Fig. 14. Both methods show that the distribution of events is 


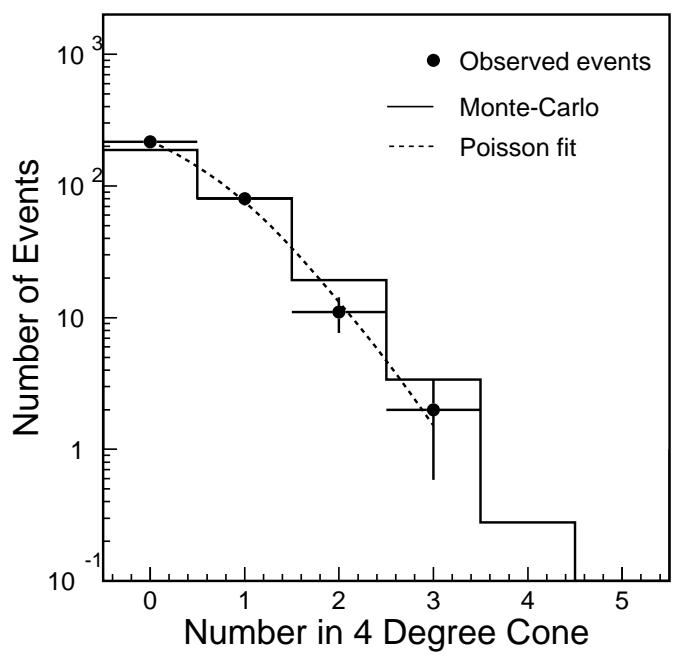

Fig. 14. Number of events observed within a cone of half-angle $4^{\circ}$ of any showering muon(dots). The solid line shows the expected atmospheric neutrino background. The dashed line shows a Poisson fit to the observed number of events with mean equal to 0.35 events

consistent with the null hypothesis of having observed no signal from unknown point sources.

\subsection{Diffuse Searches from the Galactic Plane}

Although all the expected cosmic ray-induced neutrinos observed at Super-K arise from interactions in the Earth's atmosphere, some neutrinos could be produced from collisions of cosmic rays with hydrogen from the interstellar medium $[55,56,57,58,59]$. At low energies the expected flux from these neutrinos is negligible compared to atmospheric neutrinos. According to some models [57], the minimum energy at which the atmospheric neutrino flux is equal to the flux of neutrinos produced from cosmic ray interactions in the ISM is about $10 \mathrm{TeV}$. Since most of the hydrogen is concentrated in the galactic plane, a signature of such events would be an excess from the galactic plane, which is characterized by galactic latitude of $0^{\circ}$. Searches for diffuse neutrinos from the galactic plane have been done with Super-K using all upward through-going muons [8] and with AMANDA-II [60]. We now repeat this search with the showering muon dataset.

The galactic coordinate distribution of upward showering muons is shown in Fig. 15. As in Ref. [8], we looked for a statistically significant excess within $\pm 10^{\circ}$ of the galactic plane. The observed signal events of 37 is consistent with the number of background events of 35, implying that there is no excess near galactic latitude of $0^{\circ}$. Thus we do not see any evidence that any of the upward 


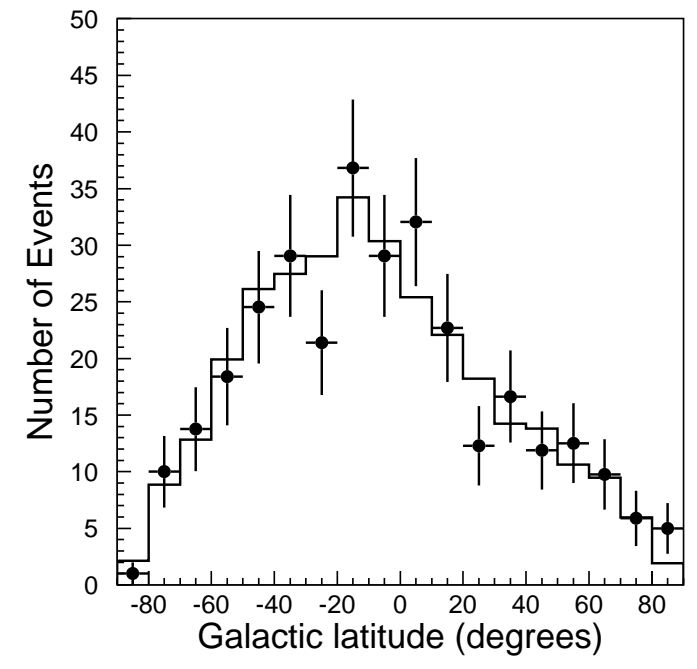

Fig. 15. Galactic latitude distribution of upward showering muons (dots) compared with Monte Carlo background from atmospheric neutrinos (solid line).

showering muon events are coming from interaction products of cosmic rays with the interstellar medium. 
Table 3. Table of observed number of upward showering muons and expected background from atmospheric neutrinos in a cone of half-angle $3^{\circ}$ from selected point sources along with $90 \%$ c.l. muon and neutrino flux limits for a $E^{-2}$ neutrino spectrum.

\begin{tabular}{|c|c|c|c|c|}
\hline Source & Data & Background & $\begin{array}{c}\mu \text { flux limits } \\
\left(10^{-15} \mathrm{~cm}^{-2} \mathrm{~s}^{-1}\right)\end{array}$ & $\begin{array}{c}\nu \text { flux limits } \\
\left(10^{-8} \mathrm{~cm}^{-2} \mathrm{~s}^{-1}\right)\end{array}$ \\
\hline SMC X-1 & 0 & 0.3 & 1.3 & 4.4 \\
\hline LMC X-2 & 0 & 0.3 & 1.3 & 4.4 \\
\hline SN 1987A & 1 & 0.3 & 2.0 & 7.0 \\
\hline LMC X-4 & 0 & 0.3 & 1.3 & 4.4 \\
\hline GX301.2 & 0 & 0.6 & 1.3 & 4.5 \\
\hline Cen X-5 & 2 & 0.4 & 2.7 & 9.6 \\
\hline GX 304-1 & 1 & 0.4 & 2.0 & 7.0 \\
\hline Cen X-3 & 0 & 0.3 & 1.3 & 4.5 \\
\hline Cir X-1 & 1 & 0.6 & 1.9 & 6.8 \\
\hline $2 \mathrm{U} 1637-53$ & 0 & 0.3 & 1.3 & 4.6 \\
\hline $4 \mathrm{U} 1608-522$ & 1 & 0.3 & 1.4 & 5.0 \\
\hline GX 339.4 & 0 & 0.3 & 1.6 & 5.6 \\
\hline Vela & 1 & 0.2 & 1.8 & 6.3 \\
\hline GX 346-7 & 0 & 0.2 & 1.7 & 6.0 \\
\hline AR X-1 & 0 & 0.2 & 1.7 & 6.1 \\
\hline SN 1006 & 1 & 0.4 & 2.8 & 9.8 \\
\hline Vela X-1 & 0 & 0.6 & 1.8 & 6.5 \\
\hline $2 \mathrm{U} 1700-37$ & 0 & 0.3 & 1.9 & 6.7 \\
\hline SGR X-4 & 1 & 0.2 & 2.0 & 7.3 \\
\hline L10 & 0 & 0.4 & 2.1 & 7.2 \\
\hline GX $1+4$ & 0 & 0.6 & 2.1 & 7.6 \\
\hline SN 1604 & 0 & 0.6 & 2.2 & 7.7 \\
\hline
\end{tabular}


Table 3-Continued

\begin{tabular}{|c|c|c|c|c|}
\hline Source & Data & Background & $\begin{array}{c}\mu \text { flux limits } \\
\left(10^{-15} \mathrm{~cm}^{-2} \mathrm{~s}^{-1}\right)\end{array}$ & $\begin{array}{l}\nu \text { flux limits } \\
\left(10^{-8} \mathrm{~cm}^{-2} s^{-1}\right)\end{array}$ \\
\hline GX 9.9 & 0 & 0.4 & 2.3 & 8.0 \\
\hline Sco X-1 & 0 & 0.4 & 2.3 & 8.0 \\
\hline Aqr X-1 & 0 & 0.2 & 2.5 & 8.8 \\
\hline $4 \mathrm{U} 336+01$ & 1 & 0.3 & 4.0 & 14.1 \\
\hline Aql X-1 & 0 & 0.3 & 2.5 & 8.9 \\
\hline $2 \mathrm{U} 1907+02$ & 0 & 0.3 & 2.6 & 9.0 \\
\hline Ser X-1 & 0 & 0.2 & 2.6 & 9.2 \\
\hline $\mathrm{SS} 433$ & 0 & 0.4 & 2.6 & 9.2 \\
\hline $2 \mathrm{U} 0613+09$ & 0 & 0.1 & 2.7 & 9.5 \\
\hline Geminga & 1 & 0.1 & 2.9 & 10.3 \\
\hline Crab & 0 & 0.3 & 3.1 & 10.9 \\
\hline $2 \mathrm{U} 035+30$ & 1 & 0.1 & 5.9 & 20.8 \\
\hline Cyg X-1 & 1 & 0.1 & 3.9 & 14.0 \\
\hline Her X-1 & 0 & 0.3 & 3.9 & 13.7 \\
\hline Mrk 421 & 0 & 0.2 & 4.2 & 14.8 \\
\hline Cyg X-2 & 0 & 0.1 & 4.2 & 14.7 \\
\hline Mrk 501 & 0 & 0.2 & 4.4 & 15.4 \\
\hline Cyg X-3 & 0 & 0.2 & 4.6 & 16.1 \\
\hline Per X-1 & 0 & 0.2 & 4.7 & 16.4 \\
\hline SGR 1806 & 0 & 0.2 & 2.2 & 7.8 \\
\hline SGR 1900 & 0 & 0.2 & 2.7 & 9.6 \\
\hline SGR 1627 & 1 & 0.3 & 2.9 & 10.1 \\
\hline
\end{tabular}


Table 3-Continued

\begin{tabular}{|c|c|c|c|c|}
\hline Source & Data & Background & $\begin{array}{c}\mu \text { flux limits } \\
\left(10^{-15} \mathrm{~cm}^{-2} s^{-1}\right)\end{array}$ & $\begin{array}{l}\nu \text { flux limits } \\
\left(10^{-8} \mathrm{~cm}^{-2} s^{-1}\right)\end{array}$ \\
\hline SGR 1801 & 0 & 0.1 & 2.2 & 7.7 \\
\hline SGR 0525 & 1 & 0.3 & 2.0 & 7.0 \\
\hline LS 5039 & 1 & 0.4 & 3.5 & 12.4 \\
\hline WR 20a & 0 & 0.4 & 1.3 & 4.5 \\
\hline 1ES $1959+650$ & 0 & 0.2 & 2.7 & 9.4 \\
\hline B1509-58 & 1 & 0.4 & 2.0 & 7.0 \\
\hline B1706-44 & 0 & 0.3 & 1.7 & 6.1 \\
\hline B1823-13 & 2 & 0.4 & 5.0 & 17.5 \\
\hline HESS J1303-631 & 1 & 0.4 & 2.0 & 6.9 \\
\hline HESS J1514-591 & 2 & 0.4 & 2.7 & 9.6 \\
\hline HESS J1614-518 & 1 & 0.3 & 1.5 & 5.2 \\
\hline HESS J1632-478 & 0 & 0.3 & 1.6 & 5.7 \\
\hline HESS J1702-420 & 1 & 0.1 & 1.8 & 6.4 \\
\hline HESS J1745-303 & 0 & 0.2 & 2.1 & 7.2 \\
\hline HESS J1804-216 & 0 & 0.3 & 2.2 & 7.7 \\
\hline HESS J1825-137 & 1 & 0.4 & 3.6 & 12.6 \\
\hline HESS J1834-087 & 1 & 0.3 & 3.8 & 13.4 \\
\hline RX J0852-4622 & 0 & 0.3 & 1.7 & 6.0 \\
\hline
\end{tabular}

\section{CONCLUSIONS}

From the sample of upward through-going muons in 1646 days of data we have isolated a sample of 318 showering muon events which lose energy through radiative processes. The mean parent neutrino energy of these events is $\simeq 1 \mathrm{TeV}$ which make this subset the highest energy neutrinos seen in Super-K. The fraction of these events is approximately one fifth of upward through-going muon sample. At these high energies, the neutrino oscillation probability (for oscillation parameters obtained from Ref. [5]) is negligible. We have verified that 
the zenith angle distribution of upward showering muons is consistent with null oscillations. This shows that the highest neutrino energy dataset (with mean parent neutrino energy of $\simeq 1 \mathrm{TeV}$, path-lengths of order $10000 \mathrm{~km}$, and $\Delta m^{2} \sim 0.0025 \mathrm{eV}^{2}$ ) is consistent with null oscillation. We also performed various searches for extra-terrestrial neutrino sources with this subset, such as from WIMP annihilations, suspected known and unknown point sources, as well as diffuse sources. We do not see any evidence for astrophysical neutrinos in the upward showering muon dataset.

\section{ACKNOWLEDGEMENTS}

We gratefully acknowledge the cooperation of the Kamioka Mining and Smelting Company. The Super-Kamiokande experiment has been built and operated from funding by the Japanese Ministry of Education, Science, Sports and Culture, the United States Department of Energy, and the U.S. National Science Foundation, with support for individual researchers from Research Corporation's Cottrell College Science Award.

\section{References}

[1] Gaisser, T.K. and Grillo, A.F. Phys. Rev. D36, 2752 (1987)

[2] Super-Kamiokande Collaboration, S. Fukuda et al., Nucl. Instru. and Methods A501, 418 (2003)

[3] Super-Kamiokande Collaboration, Y. Fukuda et al., Phys. Lett. B467, 185 (1999)

[4] Super-Kamiokande Collaboration, Y. Fukuda et al., Phys. Rev. Lett. 82, 2644 (1999).

[5] Super-Kamiokande Collaboration, Y. Ashie et al., Phys. Rev. D71, 112005 (2005)

[6] Super-Kamiokande Collaboration, J. Hosaka et al., Phys. Rev. D74, 032002 (2006)

[7] Super-Kamiokande Collaboration, S. Desai et al., Phys. Rev. D70, 083523 (2004)

[8] Super-Kamiokande Collaboration, K. Abe et al., Astrophys. J. 652, 198 (2006)

[9] Super-Kamiokande Collaboration, S. Fukuda et al., Astrophys. J. 578, 317 (2002)

[10] S. Eidelman et al., [Particle Data Group], Phys. Lett. B592, 1 (2004). 
[11] D. Groom, N.V. Mokhov, and I.S. Striganov, Atomic Data and Nuclear Data Tables, 78, 183 (2001)

[12] P. Lipari and T. Stanev, Phys. Rev. D44, 3543 (1991)

[13] S. I. Dutta et al., Phys. Rev. D63, 094020 (2001)

[14] W. Lohmann, R. Kopp, and R. Voss, CERN yellow report 85-03 (1985)

[15] A. A. Petrukhin and V. V. Shestakov, Can. J. Phys, 46, S377 (1968)

[16] R. P. Kokoulin and A. A. Petrukhin, Acta. Phys. Hung. Suppl, 4, 277 (1970)

[17] L.B. Bezrukov and E.V. Bugaev, Sov. J. of Nucl. Phys, 33, 635 (1981)

[18] MACRO Collaboration, M. Ambrosio et al., Astropart. Phys. 9, 105 (1998)

[19] S. Desai, Ph.D thesis, Boston Univ. (2004)

[20] Super-Kamiokande Collaboration, M. Swanson et al., Astrophys. J. 652, 206 (2006)

[21] M. Honda et al., Phys. Rev. D70, 043008 (2004)

[22] L.V. Volkova, Sov. J. Nucl. Phys. 31, 784 (1980)

[23] M. Honda et al., Phys. Rev. D75, 043006 (2007)

[24] T.K. Gaisser, astro-ph/0612274 (2006)

[25] G.D. Barr et al., Phys. Rev. D74, 094009 (2006)

[26] Y. Hayato, Nucl. Phys. Proc. Suppl. 112, 171 (2002)

[27] Super-Kamiokande Collaboration, Y. Ashie et al., Phys. Rev. Lett. 93, 101801 (2004)

[28] G.L. Fogli et al., Phys. Rev. D66, 053010 (2002)

[29] R. Gandhi et al., Astropart. Phys. 5, 81 (1996)

[30] H. Nunokawa, O.L.G. Peres, and R. Zukanovich Funchal, Phys. Lett. B562, $279(2003)$

[31] S. Choubey, arXiv:0710.1557 (2007)

[32] J. Learned and K. Mannheim, Ann. Rev. of Nucl. and Part. Science, 50, 679 (2000)

[33] F. Halzen and D. Hooper, Rept. Prog. Phys. 65, 1025 (2002)

[34] A.M. Stasto, Int. J. Mod. Phys. A19, 317 (2004)

[35] J. Becker, arXiv:0710.1557 (2007)

[36] G. Jungman and M. Kamionkowski, Phys. Rev. D51, 328 (1995) 
[37] G. Jungman, M. Kamionkowski, and K. Griest, Phys. Rept. 267, 195 (1996)

[38] P. Gondolo and J. Silk, Phys. Rev. Lett., 83, 1719 (1999)

[39] J. Edsjo, Nucl. Phys. Proc. Suppl. 43, 265 (1995)

[40] M. Cirelli et al., Nucl. Phys. B727, 99 (2005)

[41] V. Barger et al., arXiv:0708.1325 (2007)

[42] M. Blennow, J. Edsjo, and T. Ohlsson, arXiv:0709.3898 (2007)

[43] AMANDA Collaboration, A. Achterberg et al., Astropart.Phys. 26, 129 (2006)

[44] Baikal Collaboration, V. Aynutdinov et al, Nucl. Instrum. Meth. A567, 423 (2006)

[45] AMANDA Collaboration, M. Ackermann et al., Astropart.Phys. 24, 459 (2006)

[46] MACRO Collaboration, M. Ambrosio et al., Astrophys. J. 546, 1038 (2001)

[47] T.K. Gaisser, F. Halzen, and T. Stanev, Phys. Rep. 258, 173 (1995)

[48] W. Bednarek, G.F. Burgio, and T. Montaruli, New Astron. Rev. 49, 1 (2005)

[49] D. Horan and T.C. Weekes, New Astron. Rev. 48, 527 (2004)

[50] C. Caso et al., Review of Particle Phys., Eur. J Phys. C3, 1 (1998)

[51] M.D. Kistler and J.F. Beacom, Phys. Rev. D74, 063007 (2006)

[52] A. Kappes et al., Astrophys. J. 656, 870 (2007)

[53] AMANDA Collaboration, M. Ackermann et al., Phys. Rev. D71, 077102 (2005)

[54] IceCube Collaboration, A. Achterberg et al., astro-ph/0611063 (2006)

[55] F. Stecker, Astrophys. J. 228, 919 (1979)

[56] V. Berezinsky et al., Astropart. Phys. 1, 281 (1993)

[57] G. Ingelman and M. Thunman, hep-ph/9604286 (1996)

[58] J. Candia, JCAP, 0511, 002 (2005)

[59] C. Evoli, D. Grasso, and L. Maccione, astro-ph/0701856 (2007)

[60] IceCube Collaboration, J.L. Kelley, astro-ph/0509546 (2005) 